\title{
AVALIAÇÃO ANTROPOMÉTRICA DE ESCOLARES DA REDE MUNICIPAL DE ENSINO DE FRANCISCO BELTRÃO, PARANÁ
}

\author{
Aline Cristiane Fochi ${ }^{1}$ \\ Eduardo Henrique Szpak Gaievski ${ }^{2}$ \\ Kérley Braga Pereira Bento Casaril ${ }^{3}$
}

\begin{abstract}
Resumo: A transição nutricional é caracterizada por mudanças nos padrões nutricionais de toda a população mundial. Nas últimas décadas a população vem enfrentando essa transição passando da desnutrição para a prevalência de sobrepeso e de obesidade. A avaliação antropométrica é um dos métodos mais utilizados para identificar alterações no estado nutricional e estabelecer intervenções preventivas elou curativas. O presente estudo teve como objetivo avaliar o estado nutricional de escolares de cinco a dez anos de idade da rede municipal de ensino de Francisco Beltrão/PR e apontar riscos nutricionais, por meio da avalição antropométrica. Os parâmetros avaliados foram: estatura para a idade (E/I), peso para a idade (P/I) e indice de massa corporal para a idade (IMC/I). A população em estudo demonstrou normalidade nos índices avaliados, com pesos e estaturas adequadas para a idade, todavia percebe-se que os níveis de sobrepeso e obesidade são bem significativos e elevados, bem como apresenta dados ainda relevantes de desnutrição. Assim, os dados revelam que embora a população estudada já esteja passando pela transição nutricional, ainda não resolveu os problemas decorrentes da desnutrição infantil. Palavras-chave: Escolares. Estado nutricional. Transição nutricional. Índices antropométricos.
\end{abstract}

\section{ANTHROPOMETRIC EVALUATION OF SCHOOLCHILDREN FROM THE NETWORK MUNICIPAL TEACHING OF FRANCISCO BELTRÃO, PARANÁ}

\begin{abstract}
The nutritional transition is characterized by changes in the nutritional patterns of the entire world population. In recent decades, the population has been experiencing this transition, going from malnutrition to the prevalence of overweight and obesity. Anthropometric assessment is one of the most used methods to identify changes in nutritional status and to establish preventive and/or curative interventions. The present study aimed to evaluate the nutritional status of students aged five to ten years old from the network municipal teaching of Francisco Beltrão/PR and point out nutritional risks by means of anthropometric assessment. The parameters evaluated were: height for age (H/A), weight for age (W/A) and body mass index for age (BMI/A). The study population showed normality in indices evaluated, with weights and height appropriate for age, however it is perceived that the levels of overweight and obesity are significant and high, as well as presenting worrying data of malnutrition. Thus, the data reveal that although the population studied is already undergoing nutritional transition, it has not yet solved the problems arising from child malnutrition.
\end{abstract}

Keywords: Schoolchildren. Nutritional state. Nutritional transition. Anthropometric indices.

\section{Introdução}

A idade escolar, entre os seis e os dez anos de idade, é um período caracterizado por um ritmo de crescimento mais acelerado. Nessa faixa etária, as crianças apresentam maiores necessidades nutricionais, têm um rápido ganho de peso em curto período, em consequência da proximidade ao estirão de crescimento da adolescência. É nessa fase que iniciam novas relações sociais, no qual novos hábitos de vida passam a se desenvolver, sendo crucial as escolhas alimentares, podendo ou não determinar a ocorrência de sobrepeso ou de obesidade. Nesse sentido, as crianças são consideradas importantes grupos-alvo para investigação de distúrbios

\footnotetext{
${ }^{1}$ Graduada em Nutrição pela Universidade Estadual do Oeste do Paraná - Unioeste, Campus de Francisco Beltrão. E-mail: alynefocchi@hotmail.com

${ }^{2}$ Mestre em Ciência dos Alimentos pela Universidade de São Paulo - USP. E-mail: eduhsg @ gmail.com

${ }^{3}$ Doutora em Ciência de Alimentos. Líder do Grupo de Estudo e de Pesquisa em Segurança Alimentar - GEPSA. Professora Associada da Universidade Estadual do Oeste do Paraná - UNIOESTE, no Programa de Pós-Graduação em Ciências Aplicadas à Saúde - PPGCAS. E-mail: kcasaril@gmail.com
} 
nutricionais, apresentando grandes possibilidades de sucesso das ações a serem implementadas, sendo o ambiente escolar ideal para esta prática (GRILLO et al., 2016).

Em todo o mundo, cresce a preocupação com a alimentação e a nutrição, especialmente na infância, pois é nesse período da vida que as pessoas estão mais expostas ao risco nutricional, mais predispostos a desenvolverem obesidade e outras doenças recorrentes (COLEONE et al., 2017). Os órgãos competentes estão se mobilizando para formar indivíduos com uma nutrição adequada, evitando que problemas nutricionais possam ocorrer na população futura (ZANCUL e FABBRO, 2007).

A transição nutricional vem sendo enfrentada em todo o mundo, em todas as faixas etárias e classes sociais, caracterizada por modificações no perfil nutricional da população, passando da desnutrição para a obesidade (RODRIGUES et al., 2011). As pesquisas brasileiras vêm demonstrando essas mudanças, como a Pesquisa de Orçamentos Familiares (POF) e a Vigilância de Fatores de Risco e Proteção para Doenças Crônicas por Inquérito Telefônico do ano de 2009, revelando altos índices de sobrepeso e de obesidade (BRASIL - IBGE, 2010; VIGITEL - BRASIL, 2013).

Segundo a Organização Mundial da Saúde (OMS), os índices de sobrepeso em crianças vêm aumentando gradativamente, sendo considerada um problema de Saúde Pública. Por isso torna-se importante que intervenções sejam realizadas, antes mesmo dos dez anos de idade pois, têm maior sucesso, sabendo-se que o controle da obesidade em adultos é pouco eficaz, tornandose necessário identificar crianças de risco para o controle do problema nutricional, evitando maiores consequências na vida adulta (BARRETO; BRASIL e MARANHÃO, 2007; FAGUNDES et al., 2008).

As pesquisas apontam que no Brasil, as taxas de excesso de peso e de obesidade vêm aumentando. Estudo realizado por Leal et al. (2012) na Região Sul do Brasil envolvendo crianças de cinco a nove anos de idade apontaram que 36,3\% dessas apresentaram excesso de peso, outros $16,7 \%$ obesidade e apenas $2,5 \%$ tem déficit de peso.

Cabe destacar que, assim como na maioria dos demais países, a obesidade é um problema de saúde pública e apresenta causas multifatoriais, envolvendo sedentarismo, fatores biológicos, comportamentais e ambientais, hábitos alimentares inadequados, ou seja, alimentos de baixo valor nutricional e de alto valor calórico são as principais causas da doença, sendo ela caracterizada pelo acúmulo de gordura corporal em excesso (DALGÊ, 2014). Crianças com excesso de peso apresentam maior risco de desenvolverem doenças cardiovasculares de maneira precoce como hipertensão arterial, dislipidemias e diabetes mellitus, antes mesmo de atingirem a vida adulta (MONEGO e JARDIM, 2006).

Devido à comprovação de que o déficit ou excesso de peso trazem riscos à saúde, a 
avaliação do estado nutricional torna-se indispensável para o acompanhamento do estado de saúde da criança, podendo ser realizado a identificação de indivíduos com alterações nutricionais, a fim de estabelecer estratégias para a redução de agravos à saúde. (DANELON, 2007; SALOMONS; RECH e LOCH, 2007).

Um método bastante utilizado na avaliação nutricional é a antropometria definida como a medida das dimensões corpóreas (VANNUCCHI et al., 1996). Essas medidas são de grande importância para a avaliação do estado nutricional dos indivíduos, pois são capazes de determinar a composição dos dois compartimentos de massa corporal: a massa magra e a massa gorda representada pelo tecido adiposo (FONTOURA et al., 2006).

Nas escolas a antropometria é muito empregada, sendo uma das formas mais utilizadas na infância para a detecção do estado nutricional, podendo ser realizados diagnósticos precoces e a partir disto, estabelecer ações educativas. Além disso, ela pode avaliar a saúde, a presença de riscos nutricionais, estabelecer a magnitude do problema, sendo de fácil operacionalização, baixo custo, método não invasivo, com objetividade e sensibilidade (ARAÚJO e CAMPOS, 2008; PEREIRA; LANZILLOTTI e SOARES, 2010; ROSSETTI; STOLARSKI e SCHIMIDT, 2014).

Para determinar o diagnóstico nutricional de crianças de 0 a 18 anos de idade de ambos os sexos, pode ser utilizado o padrão de referência mundial do Centers for Disease Control and Prevention (CDC), o qual é baseado em curvas de crescimento relacionando idade, sexo, índices de Circunferência cefálica/idade (CC/I), peso por idade (P/I), comprimento/idade (C/I), altura/idade (A/I), comprimento/altura (C/A), peso/altura (P/A) e Índice de Massa Corporal por idade (IMC/I), sendo estas essenciais para a determinação do estado nutricional, estabelecidas por valores de escore-Z ou percentis (SOARES, 2003).

Diante do exposto, a presente pesquisa tem como objetivo realizar a avaliação antropométrica de escolares de cinco a dez anos de idade da rede municipal de ensino de Francisco Beltrão/PR por meio de medidas de peso e altura, classificar o estado nutricional de cada indivíduo, observar as alterações nutricionais por faixa etária e sexo e diagnosticar casos de desnutrição, sobrepeso e obesidade entre os escolares.

\section{Metodologia}

O presente trabalho trata-se de um estudo descritivo e transversal, pesquisa de campo com abordagem quantitativa, o qual foi analisado e aprovado pelo Comitê de Ética da Universidade Estadual do Oeste do Paraná, Campus de Francisco Beltrão.

A pesquisa foi realizada em agosto de 2018, em três escolas públicas da rede municipal de ensino, sediadas em Francisco Beltrão e foi constituída por 347 escolares de cinco a dez anos de idade que se encontram matriculados nessas escolas, do $1^{\circ}$ ao $5^{\circ}$ ano. 
Os dados foram coletados por meio do preenchimento de um formulário contendo o nome do aluno, a série, a data de nascimento, o peso corporal e a estatura. Além disso, a pesquisa foi estratificada entre os sexos e as faixas etárias.

Para a aferição do peso corporal foi utilizado uma balança digital eletrônica com capacidade para 150 quilogramas e precisão de 100 gramas da marca Cadence e para obtenção da estatura dos escolares fez-se uso de uma fita métrica de 150 centímetros de extensão e subdividida em milímetros fixada na parede, onde os mesmos encontravam-se sem calçados e utilizando roupas leves para assim identificar desequilíbrios nutricionais.

Após a aferição de peso e estatura, os alunos foram classificados de acordo com seu estado nutricional. Para tanto, a avaliação dos dados antropométricos das crianças dos cinco aos dez anos de idade foi realizado utilizando o software WHO Anthro Plus, 2009 versão 1.0.3 (OMS, 2009) que determina os percentis e os escores $\mathrm{Z}$ para as relações peso para idade (P/I), estatura para idade (E/I) e índice de massa corporal para idade (IMC/I). Neste estudo, para a classificação antropométrica do estado nutricional foi adotado o escore $\mathrm{Z}$, padrão de referência da Organização Mundial da Saúde (WHO, 2006), comparando os índices de P/I, E/I e IMC/I.

A eutrofia foi definida entre os pontos de corte do escore $\mathrm{Z}$ para IMC/I +1,00 a $-2,00$; magreza acentuada abaixo de -3,00; magreza entre $-2,00$ a $-3,00$. O risco de sobrepeso definiu-se entre $+1,00$ e $+2,00$, obesidade de $+2,00$ a $+3,00$ e obesidade grave acima do escore $Z+3,00$. Para P/I, as crianças com escore Z entre -3,00 e -2,00 foram classificadas com baixo peso, escore $Z$ entre $-2,00$ e $+2,00$ com peso adequado para idade e escore $Z>+2,00$ com peso elevado para a idade. Já para E/I, as crianças com escore $Z$ entre -3,00 e -2,00 foram classificadas com baixa estatura para a idade, escore $\mathrm{Z}$ maior e igual que -2,00 com estatura adequado para idade (SOCIEDADE BRASILEIRA DE PEDIATRIA, 2009).

Participaram da pesquisa os alunos na faixa etária de cinco aos dez anos de idade, de ambos os sexos, matriculados em escolas públicas da rede municipal de ensino, que frequentam a rede municipal de ensino do município de Francisco Beltrão, PR, cujos pais autorizaram, por meio do Termo de Compromisso Livre e Esclarecido, a participarem do estudo.

Foram excluídos do estudo, aqueles alunos cujos pais não autorizarem a participar, não fizeram parte da faixa etária de 5 a 10 anos e que possuíam alguma deficiência física do tipo grave, ou que não se encaixassem de alguma forma na avaliação dos parâmetros antropométricos.

Por tratar-se de uma pesquisa não probabilística, as crianças só participaram do estudo perante assinatura dos pais ou responsáveis de Termo de Compromisso Livre e Esclarecido (TCLE). Para tanto, foram seguidos todos os preceitos éticos estabelecidos pela Resolução $\mathrm{n}^{\circ}$ 466/2012, do Conselho Nacional de Saúde (BRASIL, 2012) e o projeto de pesquisa foi 
submetido ao Comitê de Ética envolvendo seres humanos para atender normas vigentes atuais e aprovado.

\section{Resultados e Discussões}

Foram avaliadas 347 crianças matriculadas em três escolas da rede municipal de ensino de Francisco Beltrão/PR, as quais foram autorizadas pelos pais e/ou responsáveis a participar do estudo. Todas as crianças tiveram seu peso aferido, bem como foram feitas medidas para determinar o comprimento/altura. Posteriormente, conforme a faixa etária, as crianças foram classificadas quanto ao peso para idade (P/I), estatura para idade (E/I) e índice de massa corporal para idade (IMC/I) de acordo com as curvas da Organização Mundial da Saúde (OMS).

De modo geral, a figura 1 apresenta os resultados dos índices avaliados na população estudada. Observa-se que quanto ao índice E/I, do total de alunos avaliados, 98,5\% ( $\mathrm{n}=342)$ apresentaram estatura adequada para a idade e 1,4\% $(n=5)$ baixa estatura para a idade. Em relação ao índice P/I, 83,3\% $(\mathrm{n}=289)$ das crianças avaliadas apresentaram peso adequada para idade, $14,1 \%(\mathrm{n}=49)$ foram classificadas com peso elevado para a idade e 2,6\% (n=9) com baixo peso para idade. Quanto ao IMC/I das crianças avaliadas observa-se que, 59,1\% ( $\mathrm{n}=205)$ estavam eutróficas, 13,3\% ( $n=46)$ estavam em sobrepeso, 11,8\% $(n=41)$, estavam obesas, 9,2\% $(n=32)$ apresentam risco para sobrepeso, 4,6\% ( $n=16)$ estavam em estado de obesidade grave, $1,7 \%(n=6)$ magreza e $0,3 \%(n=1)$ apresentava magreza acentuada.

No cômputo geral, observa-se que a maioria das crianças se encontram tanto como o peso, bem como, com a estatura adequada para a idade. Todavia, os dados também já são bastante preocupantes, pois 40,9\% das crianças avaliadas no índice IMC/I apresenta algum risco nutricional como magreza, magreza acentuada, risco de baixo peso, além de sobrepeso, obesidade e obesidade grave.

Os dados revelam que boa parte da população estudada já apresenta problemas e consequências relacionados ao aumento de peso.

Figura 1. Perfil antropométrico de escolares com idade entre 5 anos até 10 anos de idade da rede municipal de Francisco Beltrão, segundo os indicadores peso para idade, estatura para idade e índice de massa corporal para idade, Francisco Beltrão, Paraná, 2018. 


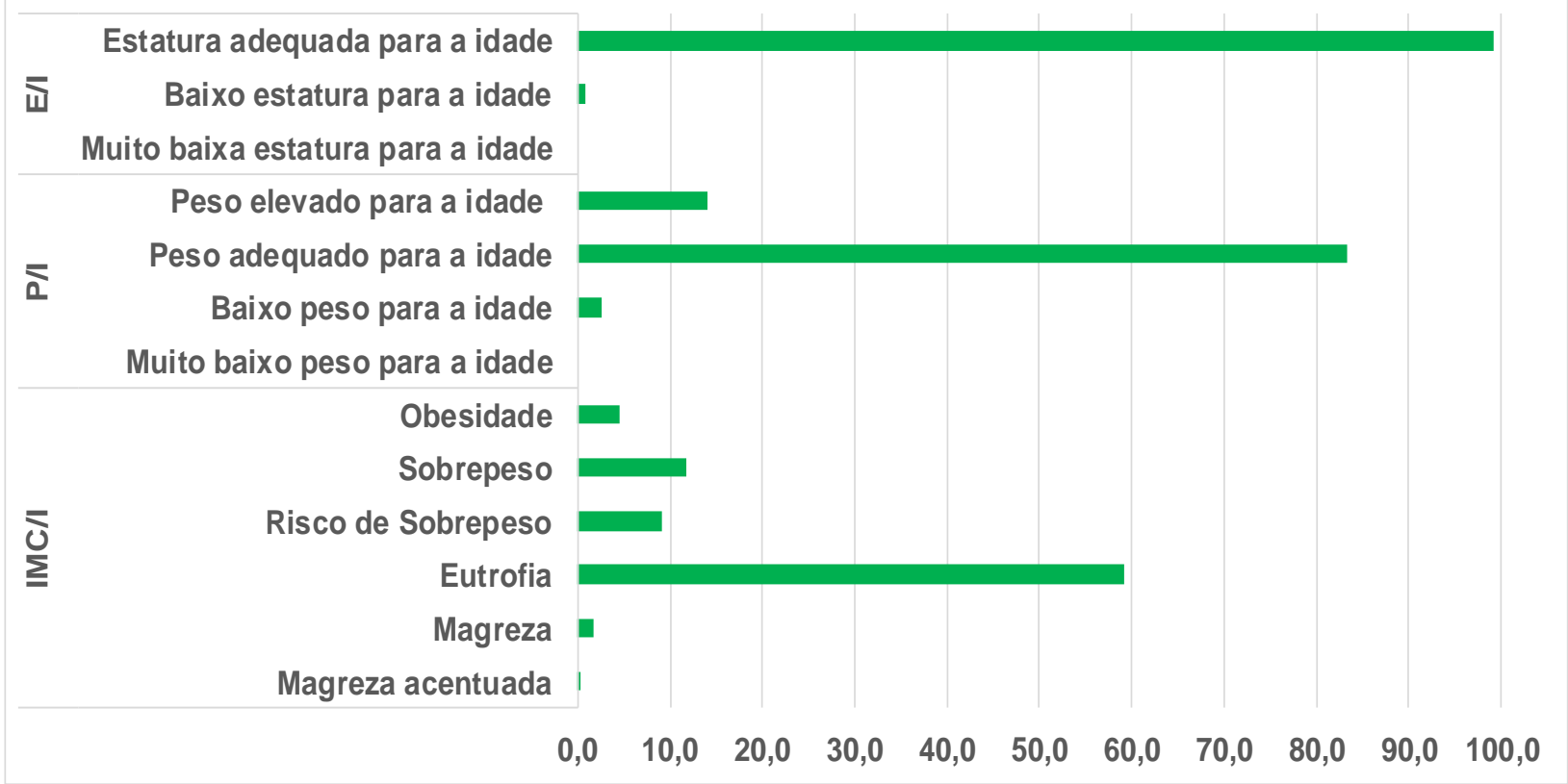

Em um estudo de Vieira et al. (2011), com 84 alunos de três a nove anos e onze meses de idade realizado no município de Pacatuba/SE, observou-se uma prevalência de eutrofia no grupo avaliado, ou seja, 89,3\% foram classificados como eutróficos segundo o IMC/I, mas em contrapartida apresentou 7,4\% de baixo peso e 3,57\% de excesso de peso, ao contrário do presente estudo que demonstrou maior relevância em casos de sobrepeso e menores índices de baixo peso. Em relação a curva de E/I pode-se observar que 92,86 \% da população de estudo encontrava-se com altura adequada para a idade o que corrobora com o presente estudo.

Na pesquisa de Brito, Walsh e Damião (2013) realizada em uma escola de Uberaba-MG para avaliar o estado nutricional de escolares de seis a dez anos de idade, nota-se uma prevalência de $99,1 \%$ de estatura adequada para a idade e déficit de estatura de $0,9 \%$. Quando avaliado o índice P/I, constatou-se que do total de escolares, 1,1\% apresentaram baixo peso para a idade, $11,8 \%$ obtiveram peso elevado para a idade e $87,1 \%$ peso adequado. Segundo o IMC/I ,14\% apresentaram obesidade ou obesidade grave, 15,5\% sobrepeso, $2 \%$ magreza e $68,5 \%$ eutrofia. Pode-se constatar que, grande parte da população estudada apresentou estatura e peso adequado para idade e eutrofia segundo o IMC, dados semelhantes foram observados na presente pesquisa.

Salomons, Rech e Loch (2007) no município de Arapoti/PR em uma avaliação com 1.647 escolares de seis a dez anos de idade, foram encontrados resultados similares com o presente estudo, onde a maior concentração de escolares foi classificada como eutrófica em ambos os sexos. Entretanto, uma proporção considerável dos escolares apresentou desnutrição (22,7\%), sobrepeso $(10 \%)$ ou obesidade $(10,9 \%)$.

No que se refere ao índice peso para idade conforme a idade escolar (Figura 2), observa- 
se que a maioria das crianças avaliadas estavam com peso adequado para a idade. A faixa etária de seis a sete anos de idade apresenta a menor porcentagem de crianças com peso elevado para a idade (4,8\%). Um estudo realizado por Moz e Santolin (2014) em Erechim/RS, com objetivo de avaliar o estado nutricional de escolares de 7 a 10 anos de idade, utilizando uma amostra de 45 estudantes, observou que $71 \%$ foram classificados com peso adequado para a idade, porém $24 \%$ dos alunos estavam com peso elevado para idade e $5 \%$ com baixo peso.

Figura 2. Perfil antropométrico das crianças com idade entre 5 a 10 anos, em três escolas da rede municipal de Francisco Beltrão segundo o indicador peso por idade, conforme a idade escolar, Francisco Beltrão, Paraná, 2018.

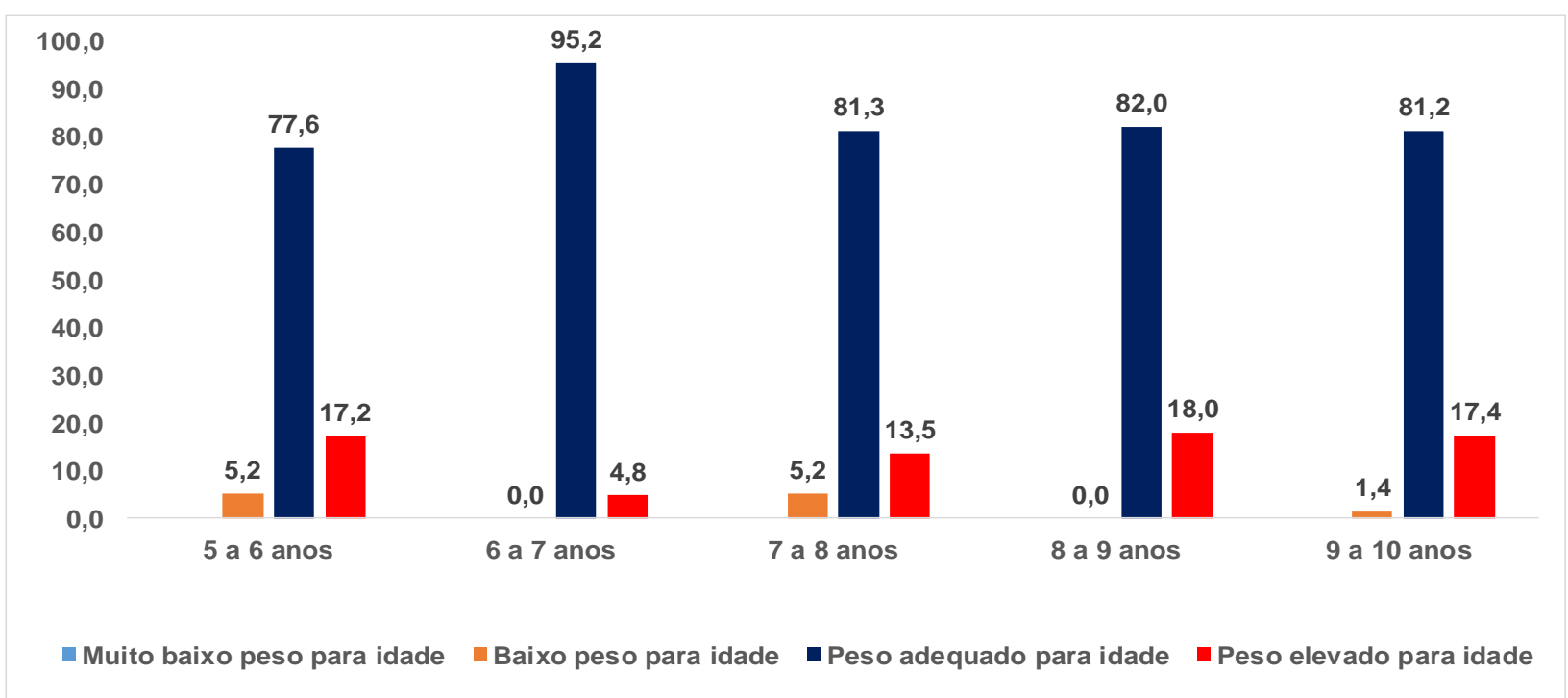

Para o índice estatura por idade, conforme a faixa etária (Figura 3) verificou-se que das crianças avaliados 98,5\% apresentam estatura adequada para a idade. A altura é um dos mais importantes parâmetros de qualidade de vida da população e o crescimento em altura de crianças apresentam como fatores determinantes o melhor acesso das crianças e seus familiares aos serviços de saúde, ao saneamento básico e melhoria nas condições de moradia, além de melhoria no nível educacional dos pais (COUTINHO; GENTIL e TORAL, 2008). Estudo desenvolvido por Bezerra et al. (2011) em Vera Mendes/PI com 60 escolares de uma escola da rede municipal de ensino observou-se que, $87,5 \%$ dos escolares apresentaram estatura adequada para idade e $12,5 \%$ encontravam-se com baixa estatura.

Figura 3. Perfil antropométrico das crianças com idade entre 5 a 10 anos, em três escolas da rede municipal de Francisco Beltrão segundo o indicador estatura para idade, conforme a idade, Francisco Beltrão, Paraná, 2018. 


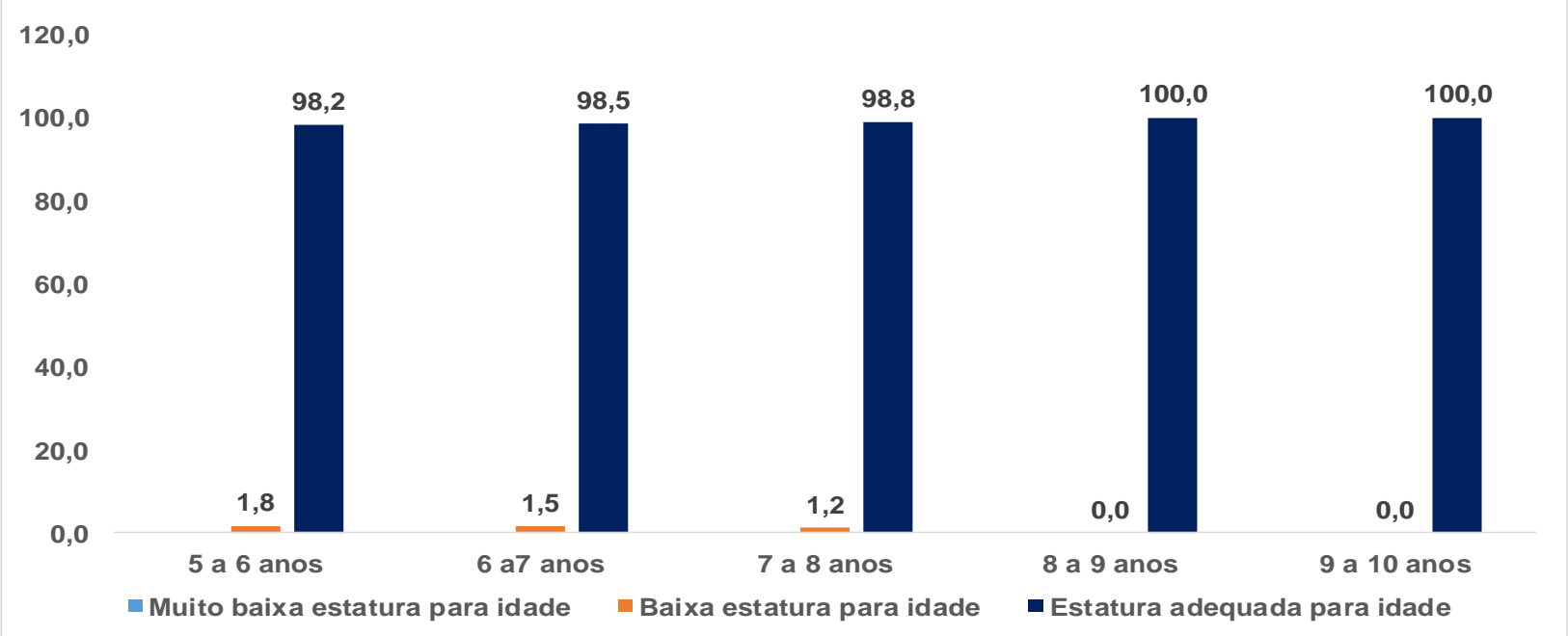

Em relação ao índice de massa corporal para idade (IMC/I), conforme a faixa etária verificou-se que do total de crianças avaliadas, 59,1\% encontram-se em eutrofia, no entanto, pode-se observar na figura 4 que à medida que as crianças vão ficando mais velhas o risco nutricional relacionado ao aumento de peso vai aumentando.

De maneira geral, pode-se perceber um aumento no número de casos de sobrepeso e obesidade à medida que as crianças vão envelhecendo. Dos 5 aos 6 anos de idade $15 \%$ apresentam risco de sobrepeso, 13,5\% sobrepeso, 1,9\% obesidade e 7,7\% obesidade grave, já na faixa etária dos 6 aos 7 anos 9,7\%, 6,5\%, 1,6\% e 1,6\% respectivamente. A partir dos 7 anos de idade esses números aumentaram gradativamente, onde dos 7 aos 8 anos, 8,5\% estavam em risco de sobrepeso, $9,8 \%$ sobrepeso, $9,8 \%$ obesidade e $3,7 \%$ obesidade grave, além disso dos 8 aos 9 anos de idade, $8,3 \%, 9,7 \%, 22,2 \%$ e 1,4\% respectivamente. A maior preocupação se dá na faixa etária dos 9 aos 10 anos de idade, no qual 6,3\% apresentavam risco de sobrepeso, 25,3\% em sobrepeso, $19,0 \%$ obesidade e $8,9 \%$ em obesidade grave.

Figura 4. Perfil antropométrico das crianças com idade entre 5 a 10 anos, em três escolas da rede municipal de Francisco Beltrão segundo o indicador índice de massa corporal para idade, conforme a faixa etária, Francisco Beltrão, Paraná, 2018. 


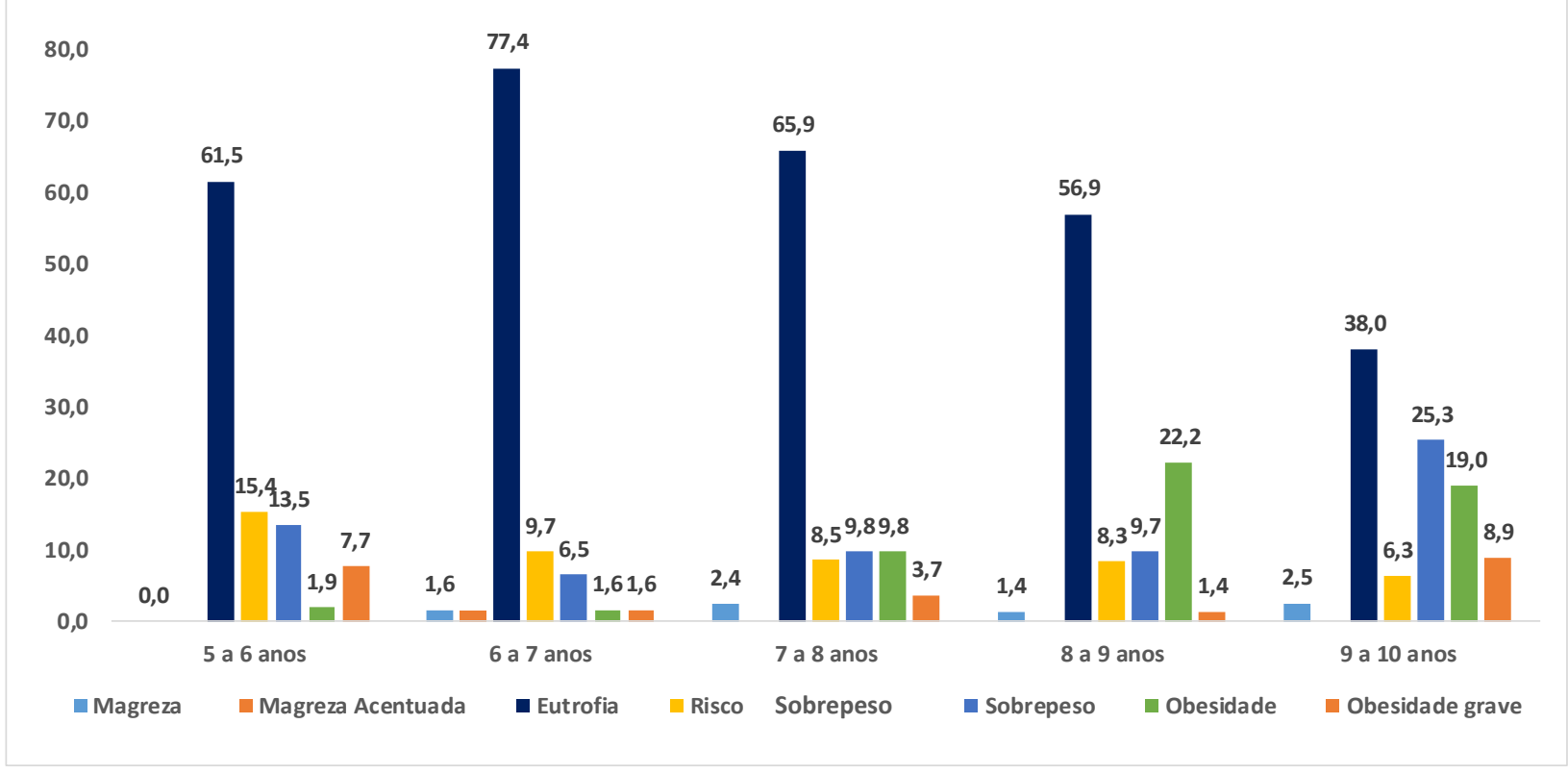

Este dado é bastante evidente na faixa etária dos nove a dez anos de idade, na qual se observa um número de indivíduos eutróficos relativamente menor (38\%) quando comparada às outras idades, consequentemente o sobrepeso, obesidade e obesidade grave nessa faixa etária foram elevados, situações de magreza e magreza acentuadas foram considerados baixos e aceitáveis pela OMS que recomenda valores menores que 5\%. Também, pode ser observado uma prevalência de obesidade dos oito aos nove anos de idade. Contudo, é possível comprovar a transição nutricional que a população vem enfrentando, passando da desnutrição para sobrepeso e obesidade.

Esses resultados são semelhantes aos observados por Moz e Santolin (2014) no município de Erechim/RS com avaliação de 45 escolares dos 7 aos 10 anos de idade, os quais observaram que $53 \%$ dos escolares encontravam-se em eutrofia, enquanto, $45 \%$ apresentaram algum risco nutricional, ou seja, em sobrepeso ou obesidade.

Jesus e Simões (2011), em um estudo com 261 escolares, relatam uma situação similar, em que $65,9 \%$ dos escolares são eutróficos, seguidos de 15,7\% com sobrepeso, $12,3 \%$ obesos, $5,4 \%$ obesos graves e sendo apenas $0,4 \%$ que apresentaram magreza e $0,4 \%$ magreza acentuada.

Em um estudo realizado em Florianópolis/SC com escolares de 7 a 10 anos de idade ressalta os casos aumentados de sobrepeso e obesidade, $17,1 \%$ e 8,2\% respectivamente, comprovando que a medida que as crianças envelhecem, elas vão ganhando peso de forma descontrolada, como mostra o presente estudo contendo resultados semelhantes (FORTE, 2003).

Os índices aumentados de sobrepeso, obesidade e obesidade grave demonstrados pelos indicadores IMC/I e P/I, podem ser justificados pela existência do estirão pubertário, que até os dez anos podem apresentar certo desvio normal do peso, sendo considerada um processo de 
repleção energética (BERGAMASCHI; ADAMI, 2015).

Ao comparar os índices utilizados, observou-se que o índice de massa corporal para idade foi mais sensível para diagnosticar excesso de peso nos escolares do presente estudo.

No Brasil, a utilização do índice peso para a idade é preconizada pelo Ministério da Saúde para avaliar o risco de sobrepeso e obesidade no âmbito individual. No entanto, as recomendações recentes do Center for Disease Control (CDC) apontam o índice de massa corporal como método para avaliar a situação de sobrepeso ou obesidade em crianças, a partir de dois anos de idade. Tal indicador teria a vantagem de incorporar o valor da estatura da criança na caracterização do estado nutricional (COLE et al., 2000).

Os dados da presente pesquisa também foram estratificados conforme o sexo dos avaliados. Por meio do quadro 01, observa-se o estado nutricional utilizando-se o indicador P/I dos escolares, sendo possível identificar que entre meninos e meninas ocorreu uma prevalência do peso adequado para idade, de $80 \%$ e $86,4 \%$ respectivamente. Porém, os casos de peso elevado para a idade demonstraram certa preocupação, com 17,6\% para o sexo masculino e 10,8\% para o feminino. No entanto, o número de indivíduos que apresentaram baixo peso para a idade foi reduzido, sendo de $2,4 \%$ para meninos e $2,8 \%$ para meninas, o que comprova a transição nutricional enfrentada atualmente.

Quadro 01. Perfil antropométrico das crianças com idade entre 5 a 10 anos, em três escolas da rede municipal de Francisco Beltrão para o indicador peso para idade, segundo o sexo, Francisco Beltrão, Paraná, 2018.

\begin{tabular}{|l|c|c|c|c|c|c|}
\hline \multirow{2}{*}{ Classificação (Peso/idade) } & \multicolumn{2}{|c|}{ Meninos } & \multicolumn{2}{c|}{ Meninas } & \multicolumn{2}{c|}{ Total } \\
\cline { 2 - 7 } & $\mathbf{N}^{\mathbf{0}}$ & $\boldsymbol{\%}$ & $\mathbf{N}^{\mathbf{o}}$ & $\boldsymbol{\%}$ & $\mathbf{N}^{\mathbf{0}}$ & $\boldsymbol{\%}$ \\
\hline Muito baixo peso para idade & 0 & 0 & 0 & 0 & 0 & 0 \\
\hline Baixo peso para idade & 4 & 2,4 & 5 & 2,8 & 9 & 2,6 \\
\hline Peso adequado para idade & 136 & 80 & 153 & 86,4 & 289 & 83,3 \\
\hline Peso elevado para idade & 30 & 17,6 & 19 & 10,8 & 49 & 14,1 \\
\hline
\end{tabular}

Estes dados se correlacionam com o estudo de Brito, Walsh e Damião (2013), que avaliou 458 alunos em idade entre 6 a 10 anos de uma escola da rede pública de ensino de Uberaba/MG, cujos dados apresentaram predomínio de peso adequado para idade, $86,5 \%$ para o sexo masculino e $87,8 \%$ para o sexo feminino. Porém, também foram registradas taxas alarmantes de peso elevado para idade para meninos e meninas, com 12,3\% e 11,2\% respectivamente.

O perfil antropométrico dos escolares foi também avaliado pelo Índice estatura para idade, demonstrado no quadro 02 , onde observou-se uma prevalência da estatura adequada para a idade, $98,2 \%$ para o sexo masculino e $98,9 \%$ para o sexo feminino. Porém, casos de baixa 
estatura para a idade podem ser observados em $1,8 \%$ nos meninos e $1,1 \%$ para as meninas, sendo considerados valores reduzidos e aceitáveis.

Quadro 02. Perfil antropométrico das crianças com idade entre 5 a 10 anos, em três escolas da rede municipal de Francisco Beltrão para o indicador estatura para idade, segundo o sexo, Francisco Beltrão, Paraná, 2018.

\begin{tabular}{|l|c|c|c|c|c|c|}
\hline \multirow{2}{*}{ Classificação (Estatura/idade) } & \multicolumn{2}{|c|}{ Meninos } & \multicolumn{2}{c|}{ Meninas } & \multicolumn{2}{c|}{ Total } \\
\cline { 2 - 7 } & $\mathbf{N}^{\mathbf{0}}$ & $\mathbf{\%}$ & $\mathbf{N}^{\mathbf{o}}$ & $\mathbf{\%}$ & $\mathbf{N}^{\mathbf{0}}$ & $\boldsymbol{\%}$ \\
\hline $\begin{array}{l}\text { Muito baixa estatura para } \\
\text { idade }\end{array}$ & 0 & 0 & 0 & 0 & 0 & 0 \\
\hline Baixa estatura para idade & 3 & 1,8 & 2 & 1,1 & 5 & 1,4 \\
\hline Estatura adequada para idade & 167 & 98,2 & 175 & 98,9 & 342 & 98,6 \\
\hline
\end{tabular}

Como observado na tabela acima, os valores de déficit de E/I para escolares dos cinco aos dez anos de idade, independentemente da idade foram de 2,8\% de ambos os sexos. Esses dados se assemelham aos observados em um estudo realizado em Pelotas/RS por Vieira et al. (2008) em um censo escolar que avaliou 2004 alunos do $1^{\circ}$ ao $4^{\circ}$ ano, onde os percentuais de baixa estatura para idade não ultrapassaram os $4 \%$.

Em relação ao indicador IMC/I segundo o sexo, pode-se perceber que os dados obtidos para os sexo masculino e feminino foram de $1,2 \%$ e $2,3 \%$ em estado de magreza, $9,4 \%$ e $9,0 \%$ em risco para baixo peso, $57,6 \%$ e $60,5 \%$ eutróficos, $11,8 \%$ e $14,7 \%$ em sobrepeso, $13,5 \%$ e $10,2 \%$ são obesos e $6,5 \%$ e $2,8 \%$ apresentaram obesidade grave, respectivamente.

Quadro 03. Perfil antropométrico das crianças com idade entre 5 a 10 anos, em três escolas da rede municipal de Francisco Beltrão para o indicador índice de massa corporal para idade, segundo o sexo, Francisco Beltrão, Paraná, 2018.

\begin{tabular}{|l|c|c|c|c|c|c|}
\hline \multirow{2}{*}{ Classificação (IMC/Idade) } & \multicolumn{2}{|c|}{ Meninos } & \multicolumn{2}{c|}{ Meninas } & \multicolumn{2}{c|}{ Total } \\
\cline { 2 - 7 } & $\mathbf{N}^{\mathbf{0}}$ & $\mathbf{\%}$ & $\mathbf{N}^{\mathbf{0}}$ & $\mathbf{\%}$ & $\mathbf{N}^{\mathbf{0}}$ & \% \\
\hline Magreza & 2 & 1,2 & 4 & 2,3 & 6 & 1,7 \\
\hline Magreza acentuada & 0 & 0 & 1 & 0,6 & 1 & 0,3 \\
\hline Risco para baixo peso & 16 & 9,4 & 16 & 9,0 & 32 & 9,2 \\
\hline Eutrofia & 98 & 57,6 & 107 & 60,5 & 205 & 59,1 \\
\hline Sobrepeso & 20 & 11,8 & 26 & 14,7 & 46 & 13,3 \\
\hline Obesidade & 23 & 13,5 & 18 & 10,2 & 41 & 11,8 \\
\hline Obesidade Grave & 11 & 6,5 & 5 & 2,8 & 16 & 4,6 \\
\hline
\end{tabular}

Segundo Giugliano e Melo (2004) em uma pesquisa realizada em Brasília/DF envolvendo escolares de 6 a 10 anos de idade de uma instituição de ensino privado, utilizando o IMC/I como indicador para diagnóstico de sobrepeso e de obesidade em escolares, foi observado a prevalência de obesidade (obesidade + obesidade grave) no sexo masculino $(5,9 \%)$, o que corrobora com este estudo, onde os índices de obesidade em meninos é mais elevado do que nas 
meninas, de $23 \%$ para $18 \%$ respectivamente, confirmando a gravidade do problema.

No presente estudo, o excesso de peso (sobrepeso + obesidade) demonstrou valores mais elevados no sexo masculino (32\%), o que contradiz o estudo de Melo et al. (2016), realizado com escolares dos 5 aos 11 anos de idade da rede pública de ensino de Rio Branco/RO, no qual foi verificado um predomínio de excesso de peso em meninas $(27,3 \%)$.

A grande maioria dos escolares mostrou estar com peso adequado para a idade, contudo, foi possível observar valores pertinentes de pesos elevados, ressaltando maior preocupação para o sexo masculino.

A pesquisa não demonstrou diferenças em casos de excesso de peso para meninos ou meninas, porém indivíduos do sexo masculino ainda apresentaram taxas de excesso de peso um pouco aumentadas em relação ao IMC/I e P/I, que pode estar associado a uma análise realizada por Bergamaschi e Adami (2015) que apresentaram estes mesmos resultados, a qual avaliou crianças de 5 a 10 anos de idade, onde 63,9\% eram eutróficos, 27,8\% estavam em sobrepeso e $7,2 \%$ em obesidade segundo o IMC/I, nas meninas $14,25 \%$ e $1,81 \%$ estavam em sobrepeso e obesidade, já no sexo masculino $14,25 \%$ e $3,21 \%$ respectivamente, confirmando o excesso de peso nos meninos como no presente estudo.

Além disso, foi viável realizar na população em estudo a distribuição de escolares com estatura adequada ou não para cada idade, assim foi possível denotar que houve grande prevalência da estatura adequada para a idade tanto para meninos como para meninas, onde apenas $1,8 \%$ e $1,1 \%$ respectivamente, apresentaram baixa estatura para a idade.

Desta forma, apesar do elevado percentual de eutrofia nos escolares estudados, destaca-se a prevalência de excesso de peso que se distribuiu diferentemente em meninos e meninas, variando conforme a idade e menor predomínio de desnutrição.

A obesidade pode iniciar em qualquer idade, desencadeada por fatores como o desmame precoce, hábitos alimentares inadequados e sedentarismo, podendo estar relacionados ao excesso de peso e ao aumento das taxas de mortalidade (MIRANDA et al., 2015).

A obesidade infantil pode contribuir para o desenvolvimento precoce de outras doenças crônicas associadas como problemas articulares, cutâneos, gastrintestinais, respiratórios, psicossociais, interferência no crescimento e desenvolvimento da criança. As doenças endócrino metabólicas são as mais associadas a obesidade infantil, como o diabetes mellitus tipo 2, doenças cardiovasculares, com elevação da pressão arterial, High Density Lipoproteins (HDL) abaixo do recomendado, triglicerídeos elevados, resistência à insulina, que pode estar associada ao desenvolvimento da Síndrome Metabólica e o surgimento de tumores malignos (BONFIM, 2016).

Sabe-se que, a obesidade na infância tende a continuar na fase adulta, se não for 
convenientemente controlada, levando ao aumento da morbimortalidade (LEÃO et al., 2003; NASCIMENTO; MUNIZ e PINHEIRO, 2010). Assim sendo, a detecção precoce de crianças com maior risco para o desenvolvimento de obesidade, juntamente com a tomada de medidas para controlar este problema, faz com que o prognóstico seja mais favorável no longo prazo.

Os índices antropométricos são de extrema importância para identificar riscos nutricionais e classificar o estado nutricional infantil. O P/I é a relação entre a massa corporal e a idade cronológica da criança, mais sensível na identificação de baixo peso, além disso, é uma avalição muito adequada para acompanhar o ganho de peso. A E/I expressa o crescimento da criança, indicador mais sensível para verificar a qualidade de vida. Já o IMC/I é utilizado para realizar o diagnóstico individual e coletivo das alterações nutricionais (SOCIEDADE BRASILEIRA DE PEDIATRIA,2009).

\section{Considerações Finais}

A realização do presente estudo nos revela dados preocupantes, no qual muitas das crianças avaliadas se encontram em risco nutricional, principalmente pelo aumento do peso, que foi de maior relevância para o sexo masculino e na faixa etária dos 9 aos 10 anos de idade, tornando-se alarmante no cenário atual.

Vale destacar que a comparação dos dados encontradas neste estudo com aqueles observadas por outros autores torna-se complexa, tendo em vista a diversidade das metodologias aplicadas, tais como índices e pontos de corte para classificação do estado nutricional, perfil socioeconômico das crianças avaliadas, além da localização, se urbana ou rural, a disponibilidade de alimentos e o acesso à informação.

Os índices antropométricos são padrões mais utilizados para a realização dos diagnósticos nutricionais infantis, P/I é mais sensível para identificar baixo peso, E/I crescimento linear e qualidade de vida e IMC/I para identificar distúrbios nutricionais através do diagnóstico, ou seja todos esses parâmetros estão correlacionados entre si, de grande relevância para identificação do estado nutricional.

A magnitude do problema encontrada em escolares beltronenses, evidencia a necessidade de ser estabelecido intervenções, visando a mudança no estilo de vida começando desde a infância, com práticas alimentares saudáveis e realização de atividade física. E neste sentido, estudantes e profissionais de nutrição estão aptos a avaliar o estado nutricional dessas crianças, propor ações e intervenções que possam contribuir para a diminuição do risco nutricional a que estas crianças estão expostas. 


\section{Referências}

ARAÚJO A. C.; CAMPOS J. A. D. B. Subsídios para a avaliação do estado nutricional de crianças e adolescentes por meio de indicadores antropométricos. Revista Alimentação e Nutrição, v.19, n.2, p. 219-225, abr./jun. 2008.

BARRETO A. C. N. G.; BRASIL L. M. P.; MARANHÃO H. S. Sobrepeso: uma nova realidade no estado nutricional de pré-escolares de Natal, RN. Revista da Associação Médica Brasileira, v. 53, n. 5, p. 377-388, 2007.

BERGAMASCHI, D.; ADAMI, F. S. Perfil antropométrico de crianças e adolescentes. Revista Ciência e Saúde, São Luís, v. 17, n. 1, p.53-60, janeiro/ junho 2015.

BEZERRA, M. G. S.; PEREIRA T. G.; CHAGAS, L. R.; SANTOS, O. F. J.; NUNES, B. M. V. T. Perfil de saúde dos estudantes de uma rede de ensino municipal urbana. Revista de Pesquisa: Cuidado é Fundamental Online, Piauí, v. 1, n. 1, p.208-216, 2011.

BOMFIM, N. S.; GUILHERME, C. S.; SAITO, J. A; MONTEZANI, E. Obesidade infantil: principais causas e a importância da intervenção nutricional. Revista Cientifica da Escola da Saúde, Natal, v. 1, n. 5, p.31-44, 2016.

BRASIL. Ministério da Saúde. Conselho Nacional de Saúde. Resolução n. 466, de 12 de dezembro de 2012. Aprova diretrizes e normas regulamentadoras de pesquisas envolvendo seres humanos. Brasília, Diário Oficial da União, 12 dez. 2012.

BRASIL. Ministério da Saúde. IBGE - Pesquisa de Orçamentos Familiares 2008-2009. Antropometria e estado Nutricional de crianças, adolescentes e adultos no Brasil. Rio de Janeiro: [s. n.], 2010.

BRITO, J. B. S. M.; WALSH, I. A. P.; DAMIÃO, R. Estado nutricional de los estudiantes de una escuela pública estatal em Uberaba, Minas Gerais, Brasil. Revista Refacs (online), Minas Gerais, v. 1, n. 1, p.17-23, 2013.

COLE, J. T.; BELLIZZI, M. C.; FLEGAL, K. M.; DIETZ, W.H. Establishing a standard definition for child overweight and obesity worldwide: international survey. British Medical Journal, [s.1.], v. 320, n. 7244, p. 1-6, 2000.

COLEONE, J. D.; KUMPEL, D. A.; ALVES, A. L. S.; MATTOS, C. B. Perfil nutricional e alimentar de escolares matriculados em uma escola municipal. Revista Ciência e Saúde, Passo Fundo, RS, v. 10, n. 1, p.34-38, 23 fev. 2017.

COUTINHO, J. G.; GENTIL, P. C.; TORAL, N. A desnutrição e obesidade no Brasil: o enfrentamento com base na agenda única de nutrição. Cadernos de Saúde Pública, v. 24, n. 2, p. 332-340, 2008.

DALGÊ, Jéssica Jamila. Hábitos alimentares de adolescentes de uma escola de Cordeirópolis, SP. 2014. 34 f. Monografia (Especialização) - Curso de Tecnologia, Universidade Tecnológica Federal do Paraná, Medianeira, 2014.

DANELON, M. S. Estado nutricional, consumo alimentar e estilo de vida de escolares de Campinas - SP. 2007. 231f. Dissertação (Mestrado em Ciências e Tecnologia de Alimentos) Escola Superior de Agricultura "Luiz de Queiroz", Piracicaba, 2007.

FAGUNDES A. L. N.; RIBEIRO D. C.; NASPITZ L.; GARBELINI L. E. B.; VIEIRA J. K. P.; SILVA A. P.; LIMA, V. O.; FAGUNDES, D. J.; COMPRI, P. C.; JULIANO, Y. Prevalence of overweight and obesity in school children of Parelheiros region in São Paulo city, Brazil. Revista Paulista de Pediatria, v. 26, n. 3, p. 212-217, 2008.

FONTOURA, C.S.M.; CRUZ, D.O.; LONDERO, L.G.; VIEIRA, R.M. Avaliação Nutricional de Paciente Crítico. Revista Brasileira de Terapia Intensiva, Porto Alegre, v.18, n. 3, p. 298-306, 
2006.

FORTE, Cézar Figueiredo. Prevalência de sobrepeso e obesidade em crianças de 7 a 10 anos de idade, em Florianópolis. 2003. 77 f. Dissertação (Mestrado) - Curso de Ciências Médicas, Universidade Federal de Santa Catarina, Florianópolis, 2003.

GIUGLIANO, R.; MELO, A. L. P. Diagnóstico de sobrepeso e obesidade em escolares: utilização do índice de massa corporal segundo padrão internacional. Jornal de Pediatria, Rio de Janeiro, v. 80, n. 2, p.129-134, 2004.

GRILLO, L. P.; CONCEIÇÃO, M. L.; MATOS, C. H.; LACERDA, V. L. L. Estado nutricional e práticas de educação nutricional em escolares. O Mundo da Saúde, São Paulo, v. 40, n. 2, p.230-238, 2016.

JESUS, A. M.; SIMÕES, M. J. S. Avaliação antropométrica de escolares de sete a nove anos de idade da rede municipal de ensino de Mogi Guaçu, São Paulo. Revista Alimentação e Nutrição, Araraquara, v. 22, n. 2, p. 191-196, abr./jun. 2011.

LEAL, V. S.; LIRA, P. I. C.; OLIVEIRA, J. S.; MENEZES, R. C. E.; SEQUEIRA, L. A. S.; NETO, M. A. A.; ANDRADE, S. L. L. S; FILHO, M. B. Excesso de peso em crianças e adolescentes no Estado de Pernambuco, Brasil: prevalência e determinantes. Cadernos de Saúde Pública, Rio de Janeiro, v. 6, n. 28, p.1175-1182, jun. 2012.

LEÃO, L. S. C.; ARAÚJO, L; M. B.; MORAIS, L. T. L. P.; ASSIS, A. M. Prevalência de obesidade em escolares de Salvador, Bahia. Arquivos Brasileiros de Endocrinologia $e$ Metabologia, [s. 1.], v. 47, n. 2, p. 151-157, 2003.

MELO, M. E.; MIGUÉIS, G. L.; ALMEIDA, M. S.; DALAMARIA, T.; PINTO, W. J.; CUNHA, M. A.; SOUZA. O. F. Overweight and obesity of children in a brazilian elementary school: a comparison of three references. Journal ff Human Growth and Development, [s.1.], v. 26, n. 3, p.341-344, 28 nov. 2016.

MIRANDA, J. M. Q.; PALMEIRA, M. V.; POLITO, L. F. T.; BRANDÃO, M. R. F.; BOCALINI, D. S.; JUNIOR, F. J. A.; PONCIANO, K.; WISH, B. R. Prevalência de sobrepeso e obesidade infantil em instituições de ensino: públicas vs. privadas. Revista Brasileira de Medicina do Esporte, São Paulo, v. 21, n. 2, p.104-107, abril 2015.

MONEGO E. T.; JARDIM P. C. B. V. Determinantes de risco para doenças cardiovasculares em escolares. Arquivos Brasileiros de Cardiologia, v. 87, n. 1, Julho 2006.

MOZ, J. A; SANTOLIN, M. B. Avaliação do estado nutricional de crianças de 7 a 10 anos de uma escola estadual de Erechim-RS. Revista Perspectiva, Erechim, v. 38, n. 141, p.151-157, 2014.

NASCIMENTO, E.; MUNIZ, G. S.; PINHEIRO, I. L.; Evidências De transição nutricional em grupos de escolares entre 7 e 10 anos de idade na cidade de Camaragibe-PE. Revista Brasileira de Ciências da Saúde, [s. 1.], v. 14, n.2, p. 29-36.

PEREIRA A. S.; LANZILLOTTI H. S.; SOARES E. A. frequência à creche e estado nutricional de pré-escolares: uma revisão sistemática. Revista Paulista de Pediatria, São Paulo, v. 28, n. 4, p. 366-372, 2010.

RODRIGUES, P. A.; MARQUES, M. H.; CHAVES, M. G. A. M.; SOUZA, C. F.; CARVALHO, M. F. Prevalência e fatores associados a sobrepeso e obesidade em escolares da rede pública. Revista Ciência e Saúde Coletiva, Juiz de Fora, v. 16, n. 1, p.1581-1588, 2011.

ROSSETTI, F. X.; STOLARSKI, M. C.; SCHIMIDT S. T. Estado nutricional de adolescentes do Sudoeste do Paraná, Brasil. Revista Segurança Alimentar e Nutricional, Campinas, v. 21, n. 2, p. 437-447, 2014. 
SALOMONS, E.; RECH, C. R.; LOCH, M. R. Estado nutricional de escolares de seis a dez anos de idade da rede municipal de ensino de Arapoti, Paraná. Revista Brasileira de Cineantropometria e Desempenho Humano, v. 9, n. 3, p. 243-249, 2007.

SOARES, N. T. Um novo referencial antropométrico de crescimento: significados e implicações. Revista de Nutrição, Campinas, v. 16, n. 1, p.93-104, 2003.

SOCIEDADE BRASILEIRA DE PEDIATRIA. Avaliação nutricional da criança e do adolescente - Manual de Orientação. São Paulo: Sociedade Brasileira de Pediatria. Departamento de Nutrologia, 2009. 112 p.

VANNUCCHI, H.; UNAMUNO, M. R.; MARCHINI, J. S. Avaliação do estado nutricional. Revista de Medicina, Ribeirão Preto, v. 29, n. 1, p. 5-18,1996.

VIEIRA, D. A. S.; COSTA, D.; COSTA, J. O.; CURADO, F. F.; MENDES-NETTO, R. S. Socio-economical characteristics and nutritional status of children and adolescents in rural settlements in Pacatuba, Sergipe. Nutrire: Revista da Sociedade Brasileira de Alimentação e Nutrição, São Paulo, v. 36, n. 1, p. 49-69, abr. 2011.

VIEIRA, M. F. A.; ARAÚJO, C. L. P.; HALLAL, C. P.; MADRUGA, S. W.; NEUTZLING, M. B.; METIJASEVISH, A.; LEAL, C. M. A.; MENEZES, A. M. B. Estado nutricional de escolares de $1^{\circ}$ a $4^{\circ}$ séries do Ensino Fundamental das escolas urbanas da cidade de Pelotas, Rio Grande do Sul, Brasil. Cadernos de Saúde Pública, Rio de Janeiro, v. 24, n. 7, p.1667-1674, 2008.

VIGITEL - Brasil 2009 - Vigilância de fatores de risco e proteção para doenças crônicas por inquérito telefônico. Estimativa sobre Frequência e Distribuição sócio-demográfica de fatores de risco e proteção para doenças crônicas nas capitais dos vinte e seis estados brasileiros e no DistritoFederal. Brasília: Ministério da Saúde, 2013. Disponível em: http://bvsms.saude.gov.br/bvs/publicacoes/vigitel_brasil_2009.pdf

WHO AnthroPlus for personal computers Manual: Software for assessing growth of the world's children and adolescents. Geneva: WHO, 2009. Disponível em: http://www.who.int/growthref/tools/en/.

ZANCUL, M. S.; FABBRO, A. L. D. Escolhas alimentares e estado nutricional de adolescentes em escolas de ensino fundamental. Revista Alimentação e Nutrição, Araraquara, v. 18, n. 3, p.253-259, setembro 2007.

\section{Recebido em 16/06/2020 - Aprovado em 20/09/2020.}

